Original Research Paper

\title{
Efficacy of Curcumin as an Immunostimulatory Dietary Supplement for Channel Catfish
}

\author{
${ }^{1}$ Saly Hafiz, ${ }^{1}$ Kunwar K. Srivastava, ${ }^{2}$ Joseph C. Newton, \\ ${ }^{3}$ Hamed Samaha, ${ }^{4}$ Ahmed Hassan and ${ }^{1}$ Gopal Reddy \\ ${ }^{1}$ Department of Pathobiology, College Veterinary Medicine, Tuskegee University, Tuskegee, AL 36088, United States \\ ${ }^{2}$ Department of Pathology, School of Veterinary Medicine, Auburn University, Auburn, AL 36849, United States \\ ${ }^{3}$ Department of Animal Hygiene and Zoonosis, Faculty of Veterinary Medicine, Alexandria University, Alexandria, Egypt \\ ${ }^{4}$ Associate Dean, Post-graduate Studies and Research, Faculty of Veterinary Medicine, Suez Canal University, Ismailia, Egypt
}

Article history

Received: 02-06-2016

Revised: 11-11-2016

Accepted: 11-01-2016

Corresponding Author:

Saly Hafiz

Department of Pathobiology,

College Veterinary Medicine,

Tuskegee University, Tuskegee,

AL 36088, United States

Cell: (334)-333-8662

Email: saly.hafiz@gmail.com

shafiz1407@mytu.tuskegee.edu

\begin{abstract}
Natural agents such as curcumin may have the potential to enhance disease resistance in aquaculture. In this study, 30 catfish (Ictalurus punctatus), 2 years of age, were allotted to one of 3 treatment groups $(\mathrm{n}=10)$ to receive diets supplemented with $0.0,0.5$, or $1.0 \%$ curcumin. Body weights and lengths of fish were taken at $0,20,40$ and 60 days after starting the fish on experimental diets. At 60 days, blood samples were collected from 9 fish of each group and then were challenged with Aeromonas hydrophila $\left(0.1 \mathrm{~mL}\right.$ of $\left.9 \times 10^{6} \mathrm{CFU} / \mathrm{mL}\right)$ intra-peritoneally. Morbidity and mortality was observed daily for 7 days following challenge. Serum separated from blood samples was analyzed for the cytokines, interleukin-4 (IL-4) and interleukin-12 (IL-12). Results indicated significantly higher body weights and lengths but lower levels of both IL-4 and IL-12 with curcumin at 0.5 and $1.0 \%$ than the control group. At $24 \mathrm{~h}$ following challenge with A. hydrophila, mortality was $100(9 / 9), 33(3 / 9)$ and $22 \%(2 / 9)$ in the fish supplemented with $0.0,0.5$ and $1.0 \%$ curcumin, respectively. At $48 \mathrm{~h}$ following challenge, two more fish in the $0.5 \%$ group died but the fish remaining in $1.0 \%$ group survived even after 7 days. Results of this study suggest that curcumin has the potential to enhance performance of catfish and increase their disease resistance which may help in reducing use of antimicrobials in fish farming.
\end{abstract}

Keywords: Channel Catfish, Curcumin, Virulent Aeromonas hydrophila, Intraperitoneal Challenge, ELISA

\section{Introduction}

The incidence of infectious diseases combined with intensive commercial aquaculture practices are calling for additional and new strategies for disease management. The administration of antibiotics and other chemical anti-microbial agents is the most commonly used approach to control bacterial infections in fish. Recently, researchers have detected the serious threats associated with antibiotics in global public health. This effect is due to poor efficacy, potential toxicity and increased bacterial resistance from long-term usage of antimicrobial agents (Sasidharan et al., 2014). Additionally, antibiotics may also induce a negative impact in freshwater environments (Behera et al., 2010).

Alternative environment friendly, non-toxic and natural strategies that are both preventative and curative are being investigated. Natural plant extracts could be one such alternative to enhance non-specific innate immunity and resistance to diseases caused by fish pathogens such as Aeromonas hydrophila. Curcumin is an orange-yellow, hydrophobic and polyphenolic compound of turmeric (Curcuma longa) that has been shown to be an anti-inflammatory and immunomodulatory agent in fish species (Cao et al., 2015; Kang et al., 1999). However, studies depicting actual enhancement of disease resistance are lacking. The objectives of this study were to determine the potential of curcumin to enhance resistance against $A$. hydrophila infection, a Gram-negative bacterium primarily responsible for a disease known as Motile Aeromonas septicemia, or MAS (Hossain et al., 2014). More recently, MAS outbreaks are costing the commercial catfish industry millions of dollars in lost 
revenue (Hossain et al., 2013). In Southeastern states in the U.S., MAS has been especially epidemic due to the outbreak of a virulent $A$. hydrophila clonal group since 2009 (Pridgeon and Klesius, 2011; Tekedar et al., 2013).

\section{Materials and Methods}

\section{Experimental Fish}

The experimental channel catfish (Ictalurus punctatus), two years of age, were obtained from the laboratory of Dr. Joseph Newton at Auburn University College of Veterinary Medicine (Auburn, AL). Fish were acclimatized in laboratory conditions for 15 days while being fed commercial fish meal (API Pond Fish Food, Chalfont, PA) twice daily. Three groups of catfish were kept in separate 55-gallon tanks at a temperature of $27 \pm 2^{\circ} \mathrm{C}$, pH of $7.2 \pm 0.5$, dissolved $\mathrm{O}_{2}$ level of 6.6-6.9 $\mathrm{mg}$ $\mathrm{L}^{-1}$ and ammonia level of $0.01 \pm 0.005 \mathrm{mg} \mathrm{L}^{-1}$ with constant aeration. Water quality was tested using titration water test kits (API, Mars Fishc are Inc., Chalfont, PA).

\section{Curcumin}

Curcumin, marketed as CUR-500, was purchased as a powder ( $>95 \%$ purity) from UNICO Pharmaceuticals, Ludhiana, India. The 0.5 and $1.0 \%$ curcumin diets were produced by mixing 1.5 and $2.5 \mathrm{~g}$ of curcumin to 248.5 and $247.5 \mathrm{~g}$ commercial fish feeds, respectively. Afterwards, a small quantity of distilled water and commercial gelatin (Knox Original Gelatin, Lipton, Canada) were added to the mixture. The mixture was stirred into a thick paste and pushed through a manual press to produce $0.2 \mathrm{~mm}$ size pellets.

\section{Experimental Design}

Thirty fish were randomly allotted to one of three treatment groups $(n=10)$ and maintained in three 55gallon glass aquariums. To study the efficacy of curcumin on catfish growth and response to a challenge infection with Aeromonas hydrophila, the commercial feed was supplemented with $0.0,0.5$ or $1.0 \%$ curcumin and fed to the respective groups.

Body weight and length of fish were measured at 0 , 20, 40 and 60 days after starting the fish onexperimental diets. On day 60 , blood samples were collected from nine fish of each group and then were challenged with $A$. hydrophila at the infectious dose of $0.1 \mathrm{~mL}$ of $9 \times 10^{6} \mathrm{CFU} / \mathrm{mL}$ intra-peritoneally (Hossain et al., 2011). Mortality was observed daily for 7 days following the challenge. Serum samples were analyzed for the cytokines, interleukin-4 (IL-4) and interleukin-12 (IL-12) using ELISA kits.

\section{Blood and Serum Sampling}

On day 60, 9 fish from each group were anesthetized using tricaine methanesulfonate or MS-222 (Argent
Chemical Laboratories, Redmond, WA) at $150 \mathrm{mg} \mathrm{L}^{-1}$. Blood samples were drawn from the caudal vein using $1.0 \mathrm{~mL}$ syringes and 25-gauge needles. Collected samples were then transferred to regular $5 \mathrm{~mL}$ tubes and stored for $2 \mathrm{~h}$ at $4^{\circ} \mathrm{C}$. Serum samples were collected after centrifuging at $2000 \times \mathrm{g}$ for $15 \mathrm{~min}$ and stored at $-20^{\circ} \mathrm{C}$ until further analysis.

\section{Interleukin Quantification Assay}

IL-4 and IL-12 serum levels were measured using fishspecific Enzyme-Linked Immunosorbent Assay (ELISA) kits (BioSource International, Camarillo, CA) as per manufacturer's protocol. Serum Samples and standards of IL-4/IL-12 and IL-4/IL-12 HRP-labeled antibody were pipetted into Micro ELISA Strip-plate wells, which were additionally coated with purified fish cytokine antibody against IL-4/IL-12. The color change from the reaction was measured spectrophotometrically for Optical Density (O.D.) at $450 \mathrm{~nm}$ using a microplate reader (Dynatech Laboratories, Alexandria, VA). All mean O.D. values read from the spectrophotometer for four standards were used to develop a standard curve using statistical software. The concentrations of each cytokine in the serum samples, measured in $\mathrm{pg} / \mathrm{mL}$, were then determined by reading the points in the standard curve at which the O.D. values intersect with known concentration values.

\section{Statistical Analysis}

The data were statistically analyzed through GraphPad Prism 5.0 software (GraphPad Software, Inc., La Jolla, CA) and determined by one-way ANOVA. These data are shown as means \pm standard deviation. Statistical significance was considered at $p<0.05$.

\section{Results}

Effect of Curcumin Supplementation on Body Weight and Length of Fish

Results from body weights and lengths of fish measured on day 20 of the experimental period showed no significant differences between the control and curcumin supplemented fish. On days 40 and 60, fish fed with both concentrations of curcumin showed significantly higher $(\mathrm{p}<0.05)$ body weights and lengths as compared to control group of fish (Fig. 1 and 2). There were no significant differences between fish supplemented with 0.5 and $1.0 \%$ curcumin during each period measurements were done.

\section{Effect of Curcumin on Serum IL-4 and IL-12 Concentrations}

Serum from blood samples collected on day 60 before challenging the fish with $A$. hydrophila were tested for IL-4 and IL-12 concentrations. Fish 
supplemented with curcumin at 0.5 and $1.0 \%$ showed significantly $(p<0.05)$ lower levels of IL-4 (Fig. 3) as compared to the control group. However, IL-4 concentrations were similar between 0.5 and $1.0 \%$ groups. Serum levels of IL-12 (Fig. 4) were significantly lower in both curcumin-supplemented fish as compared to the control group. However, unlike IL-4, there was a significant difference in IL-12 concentration between the 0.5 and $1.0 \%$ curcumin-supplemented fish, with the $1.0 \%$ group measuring the lowest concentration of IL-12.

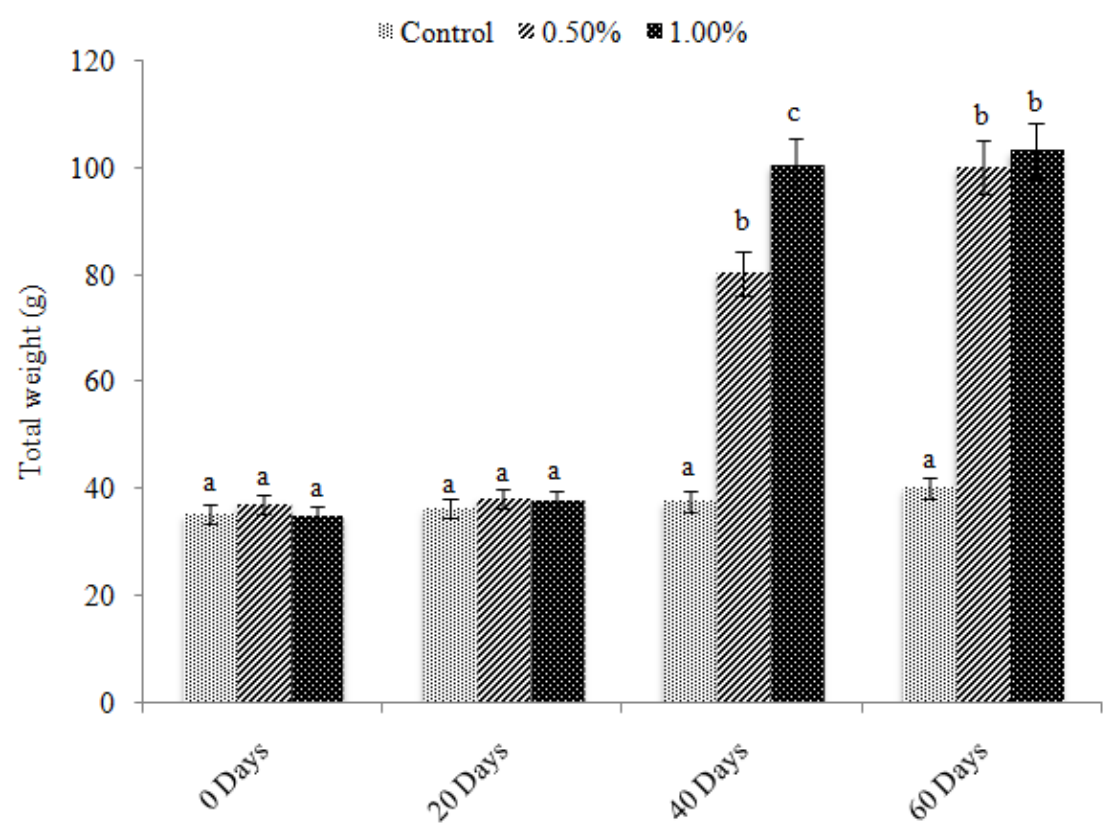

Fig. 1. Body weights of fish (in grams; means \pm standard deviation) on days $0,20,40$ and 60 . Means with different superscripts (a, b, c) denote significant differences $(p<0.05)$ between groups. On day 20 , there were no significant differences between the three groups. On day 40, significant differences appeared between the three dietary groups. At day 60, there was a significant difference between the control and curcumin-supplemented groups. However, there was no difference between the 0.50 and $1.00 \%$ groups

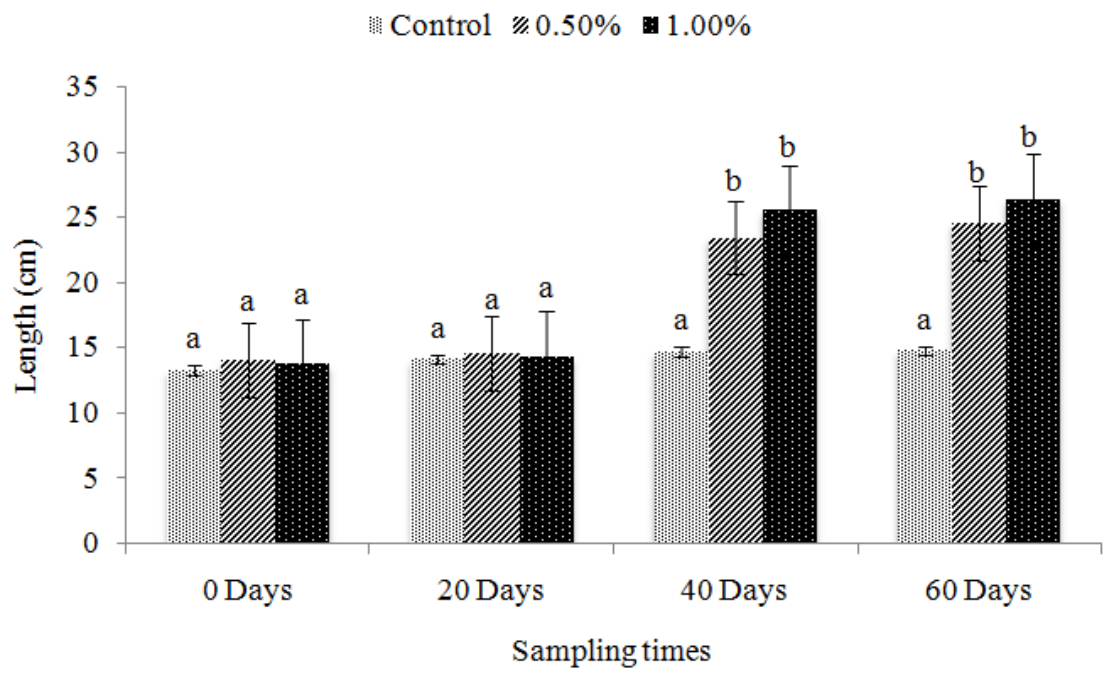

Fig. 2. Length of fish (in cm; means \pm standard deviation) measured on days $0,20,40$ and 60. Means with different superscripts (a, b, c) denote significant difference $(\mathrm{p}<0.05)$ between groups. On day 20 , there were no significant differences between the three groups. On day 40, curcumin treated groups had higher lengths as compared to control group $(\mathrm{p}<0.05)$ but there were no significant differences between two curcumin supplemented groups. On day 60, there was a significant difference between the control and curcumin-supplemented groups, however, there was no difference between the 0.5 and $1.0 \%$ groups 


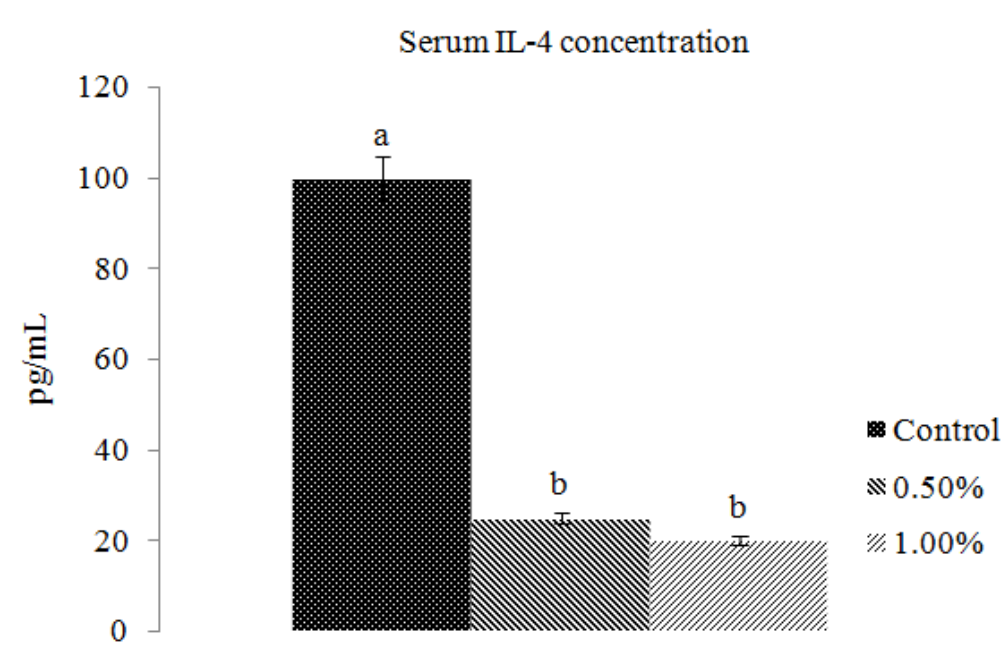

Fig. 3. IL-4 serum concentrations as measured by ELISA on day60 days of curcumin supplementation at $0.0,0.5$ and $1.0 \%$ levels. Means with different superscripts $(a, b, c)$ denote significant differences $(p<0.05)$ between groups. Concentration values, in $\mathrm{pg} / \mathrm{mL}$, are shown as means \pm standard deviation. Control group had significantly higher concentration than the two curcumin treated groups. However, there was no significant difference ( $p>0.05)$ between 0.5 and $1.0 \%$ groups

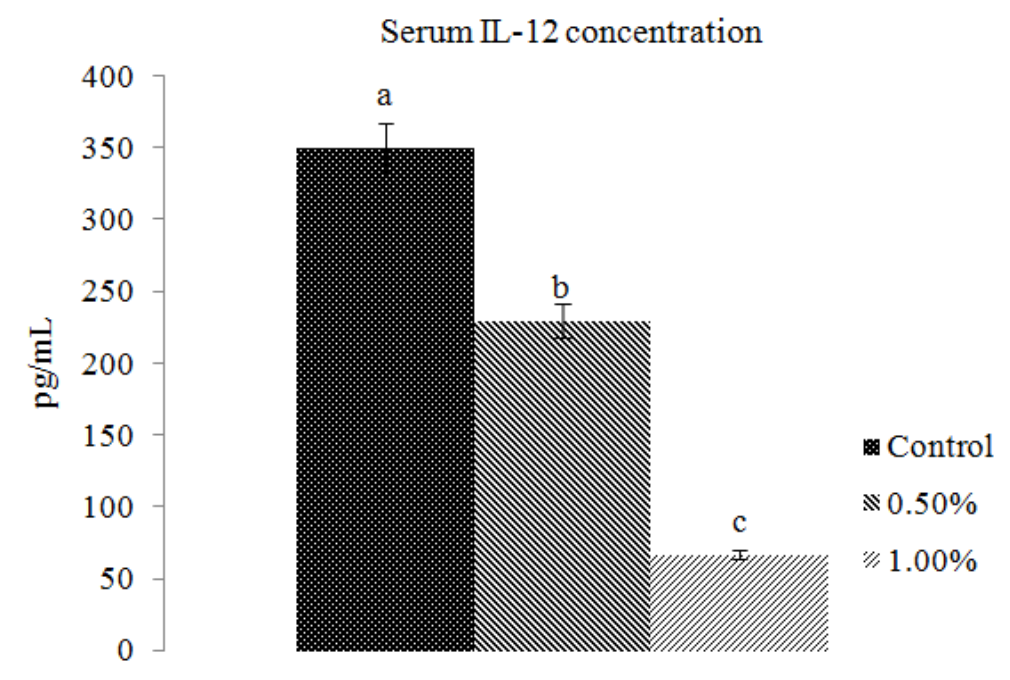

Fig. 4. IL-12 serum concentrations as measured by ELISA on day 60 of curcumin supplementation at $0.0 \%$ (control), 0.5 and $1.0 \%$ levels. Means with different superscripts $(a, b, c)$ differ significantly $(\mathrm{p}<0.05)$.Concentration values, measured in $\mathrm{pg} / \mathrm{mL}$, are shown as means \pm standard deviation. There is a significant difference between the $0.0 \% 0.5$ and $1.0 \%$ supplemented groups

\section{Effect of Curcumin on Resistance to A. hydrophila Challenge Infection}

The challenge infection of 9 fish of each group with A. hydrophila on day 60 revealed significantly lower mortality in 0.5 and $1.0 \%$ supplemented fish compared to the control group. At $24 \mathrm{~h}$ following challenge with $A$. hydrophila, $100 \%$ of the control group (9/9) died. Mortality rates significantly decreased in the 0.5 and $1.0 \%$ curcumin-supplemented groups, measuring at 33 (3/9) and $22 \%$ (2/9), respectively. At $48 \mathrm{~h}$ following challenge, two more fish in the $0.5 \%$ group died, totaling $55 \%(5 / 9)$ overall, although fish remaining in the $1 \%$ group survived even after 7 days.

\section{Discussion}

Commercial farming of Channel catfish (Ictalurus punctatus) is predominantly undertaken in the southeastern United States including State of Alabama. Farmers often face extensive economic losses due to diseases, especially due to $A$. hydrophila. There is a need for research to study the efficacy of various natural supplements that can enhance non-specific immunity against various common fish pathogens and consequently enhance growth rates. Proven efficacy of natural supplements may lead to decreased use of antibiotics and other harmful chemicals. This study attempted to determine the efficacy of curcumin, a 
natural active ingredient of Curcuma longa (turmeric), in enhancing growth rates and modulating disease resistance. Although both levels of curcumin used in this study had generally similar effects on IL-4 and IL-12 concentrations, as well as resistance against $A$. hydrophila challenge, curcumin at $1.0 \%$ level showed somewhat better efficacy. Whether supplementation at that level for longer periods will have any further beneficial effects than with $0.5 \%$ level has to be determined in future research. Physical growth parameters (length and weight) did not significantly differ between the control and curcumin-supplemented fish on day 20 of the experimental period. This was likely due to initial acclimatization needed wherein the fish adjusted to curcumin-supplemented feed. Increased growth seen at day 40 and day 60 indicated that the fish were acclimatized to the curcumin diet. Although a number of studies have reported beneficial effects of curcumin on growth rates, none have reported specific need for initial acclimatization of curcumin supplemented feed (Behera et al., 2010; Cao et al., 2015).

The significant growth response of the 0.5 and $1.0 \%$ curcumin fed groups may be attributed to increased appetite, consumption and utilization. According to Osawa et al. (1995), the enhanced growth responses indicate increased nutrient digestion and antioxidant effects of curcumin. Our results are in agreement with those of few other studies that revealed the stimulatory effects of turmeric on lipase activity, digestive enzyme activity and acid and alkaline protease activity, which all enhance the yellow pigmentation and growth rate of fish (Eumkeb et al., 2012; Kang et al., 2011). Crude protein is known to be significantly augmented while ether content decreases in fish response to turmeric supplementation (Cao et al., 2015).

The responses to challenge infection with $A$. hydrophila are probably due to the changes in performance and immunity of fish with and without curcumin-supplementation. In our studies, the challenge infection showed $100 \%$ mortality in the control group and mortality was significantly reduced in both 0.5 and $1.0 \%$ supplemented groups. These results are in accordance with (Amany Diab et al., 2014) who demonstrated a reduction in mortality of curcumin fed Oreochromis niloticus (Nile tilapia) post-challenge with Pseudomonas fluorescens.

Cytokines like IL-4 and IL-12 are regulatory proteins that are secreted by immune-response cells. Thelper 2 (Th2) cells produce cytokines, like IL-4, which play a significant role in adaptive (specific) immune response (Chen et al., 2000). IL-4 stimulates production of more Th2 cells that, in turn, produce more cytokines (Ansel et al., 2003). The Th2 mediated immunity is primarily associated with responses to pathogenic infections. It is also thought to enhance production of antibodies and inflammatory response as the cytokine is cross-linked by antigen (s) (Yates et al., 2000).
The heterodimeric cytokine IL-12 contains two subunits, p35 ( $\alpha$-chain) and p40 (a $\beta$-chain) and plays an important role in Th1 mediated immune response and inflammation. IL-12 is secreted in response to antigenic stimulation by antigen-presenting cells including macrophages, dendritic cells, human B-lymphoblastoid cells and neutrophils and has been revealed to possess cytolytic and proliferative effects of T-cells and NK cells. It may also modulate IFN $\gamma$ secretion, NK cell stimulation and maturation of cytolytic T-cells (Secombes et al., 2011). Besides these activities, the most important function of IL-12 comes from the cytokine's involvement in the progression of immature T-cells to Th1 or Th2 differentiation, thus acting as a molecular control between the activation of innate and adaptive immune responses. As such, IL-12 serves a major role in the initiation of innate (non-specific) immunity as well as the designation of Th1-immune response in higher vertebrates.

By nature of its anti-inflammatory effects, curcumin is known to inhibit various interleukins. In mice studies, IL-2 signaling in activation-induced T-lymphocytes was inhibited by curcumin, which was shown to block IL-2 production, CD25 expression and IL-2 receptor signaling (Forward et al., 2011). Another study detailed the inhibition of IL-12 synthesis by curcumin in macrophages triggered with lip opolysaccharide (Kang et al., 1999). In Nile tilapia infected with Vibrio alginolyticus, higher curcumin doses correlated with gradual but significant increase in nitric oxide production, which are involved in many critical roles in immune defense mechanisms (Elgendy et al., 2016). Nitric oxides modulate the activity of macrophages to inhibit pathogens during the process of phagocytosis (Bogdan et al., 2000).

In this study, concentrations of IL- 4 and IL-12 were lower in supplemented Channelcat fish as compared to those in the control group. Both these cytokines are known to increase in animals that are going through or have gone through episodes of infections and associated inflammations. Additionally, cytokine levels are also known to increase in animals undergoing stress due to various management practices although these changes have not been studied previously. Because of the lower levels of both IL-4 and IL-12 in curcumin supplemented fish, we conclude that supplementation with curcumin may have contributed to general non-specific immunity against infections and associated inflammation in catfish. Furthermore, our results revealing lower concentration of IL-12 due to curcumin supplementation are in accordance with similar studies (Kang et al., 1999). Together, IL-4, IL-12, IL-1 $\beta$ play an important role in innate lymphocytes in human inflammation (Bal et al., 2016). IL-12 can also stimulate similar functions to IL-2, an essential T-cell proliferation and differentiation factor in the specific immune system (Bachmann and Oxenius, 2007). 
Because curcumin supplementation has significantly enhanced protection against a challenge with $A$. hydrophila, we therefore conclude that this effect is due to enhanced non-specific immunity that may be useful in resisting other pathogens.

\section{Conclusion}

This study confirms that curcumin may possess some anti-microbial properties. Long-term studies with curcumin at $1.0 \%$ level in the diet of fish may be necessary to prove the cost-benefit ratios for the commercial fish farmers. Further studies will also be needed to analyze the molecular mechanisms by which curcumin enhances immunity in fish. As commercial kits are not available for several other important immunomodulatory cytokines and inflammatory mediators of fish, it would be necessary to study the gene expressions instead by real-time PCR.

\section{Acknowledgment}

This work was funded by U. S. Department of Agriculture (USDA), National Institute of Food \& Agriculture (NIFA), Capacity Building Grant Number: 2013-38821-21260 and USDA, NIFA Grant \#2010$38820-21475$.

\section{Author Contributions}

This article is original and does not contain already published material. The corresponding author verifies that all of the other authors have read and approved the manuscript with no ethical concerns.

\section{Conflict of Interest}

None of the authors have any conflict of interest with the work involved in this study.

\section{References}

Amany Diab, M., O. Saker, M. Eldakroury and M. Elseify, 2014. Effects of garlic (Alluim sativum) and curcumin (Turmeric, Curcuma longa Linn) on Nile tilapia immunity. Vet. Med. J. Giza, 60: 1-19.

Ansel, K.M., D.U. Lee and A. Rao, 2003. An epigenetic view of helper T cell differentiation. Nat. Immunol., 4: 616-623. DOI: 10.1038/ni0703-616

Bachmann, M.F. and A. Oxenius, 2007. Interleukin 2: From immunostimulation to immunoregulation and back again. EMBO Rep., 8: 1142-1148. DOI: 10.1038/sj.embor.7401099

Bal, S.M., J.H. Bernink, M. Nagasawa, J. Groot and M.M. Shikhagaie et al., 2016. IL-1 $\beta$, IL-4 and IL-12 control the fate of group 2 inn ate lymphoid cells in human airway inflammation in the lungs. Nat. Immunol., 17: 636-645. PMID: 27111145
Behera, T., P. Swain, S.K. Sahoo, D. Mohapatra and B.K. Das, 2010. Immunostimulatory effects of curcumin in fish, Labeo rohita (H.). Ind. J. Natural Products Resources, 2: 184-188.

Bogdan, C., M. Rollinghoff and A. Diefenbach, 2000. The role of nitric oxide in innate immunity. Immunol. Rev., 173: 17-26. DOI: $10.1034 / \mathrm{j} .1600-065 X .2000 .917307 . \mathrm{x}$

Cao, L., W. Ding, J. Du, R. Jia and Y. Liu et al., 2015. Effects of curcumin on antioxidative activities and cytokine production in Jian carp (Cyprinus carpio var. Jian) with $\mathrm{CCl}_{4}$-induced liver damage. Fish Shellfish Immunol., 43: 150-157. DOI: $10.1016 /$ j.fsi.2014.12.025

Chen, Q., N. Ghilardi, H. Wang, T. Baker and M.H. Xie et al., 2000. Development of Th1-type immune responses requires the type I cytokine receptor TCCR. Nature, 407: 916-920. PMID: 11057672

Elgendy, M., A. Hakim, T. Ibrahim, W. Soliman and S. Ali, 2016. Immunomodulatory effects of curcumin on nile tilapia, Oreochromis niloticus and its antimicrobial properties against Vibrio alginolyticus. J. Fisher. Aquatic Sci., 11: 206-215. DOI: $10.3923 /$ jfas.2016.206.215

Eumkeb, G., S. Siriwong, S. Phitaktim, N. Rojtinnakorn and S. Sakdarat, 2012. Synergistic activity and mode of action of flavonoids isolated from smaller galangal and amoxicillin combinations against amoxicillin-resistant Escherichia coli. J. Applied Microbiol., 112: 55-64. DOI: $10.1111 /$ j.1365-2672.2011.05190.x

Forward, N.A., D.M. Conrad, M.R. Power Coombs, C.D. Doucette and S.J. Furlong et al., 2011. Curcumin blocks interleukin (IL)-2 signaling in T-lymphocytes by inhibiting IL-2 synthesis, CD25 expression and IL-2 receptor signaling. Biochem. Biophys. Res. Commun., 407: 801-806. DOI: 10.1016/j.bbrc.2011.03.103

Hossain, M., Rashid, M., Sayed, M., 2011. Experimental infection of indigenous climbing perch Anabas testudineus with Aeromonas hydrophila Bacteria. Progressive Agric., 22: 105-114. DOI: $10.3329 /$ pa.v22i1-2.16472

Hossain, M.J., D. Sun, D.J. McGarey, S. Wrenn and L.M. Alexander et al., 2014. An Asian origin of virulent Aeromonas hydrophila responsible for disease epidemics in United States-farmed catfish. MBio, 5: e00848-00814. DOI: 10.1128/mBio.00848-14

Hossain, M.J., G.C. Waldbieser, D. Sun, N.K. Capps and W.B. Hemstreet et al., 2013. Implication of lateral genetic transfer in the emergence of Aeromonas hydrophila isolates of epidemic outbreaks in channel catfish. PLoS One, 8: e80943-e80943. DOI: 10.1371/journal.pone.0080943

Kang, B.Y., S.W. Chung, W. Chung, S. Im and S.Y. Hwang et al., 1999. Inhibition of interleukin-12 production in lipopolysaccharide-activated macrophages by curcumin. Eur. J. Pharmacol., 384: 191-195. DOI: 10.1016/S0014-2999(99)00690-1 
Kang, K.S., S. Yahashi, M. Azuma, A. Sakashita and S. Shioda et al., 2011. Effect of intraperitoneal injection of curcumin on food intake in a goldfish model. J. Mol. Neurosci., 45: 172-176.

DOI: $10.1007 / \mathrm{s} 12031-010-9390-5$

Osawa, T., Y. Sugiyama, M. Inayoshi and S. Kawakishi, 1995. Antioxidative Activity of Tetrahydrocurcuminoids. Biosci. Biotechnol. Biochem., 59: 1609-1612. DOI: 10.1271/bbb.59.1609

Pridgeon, J.W. and P.H. Klesius, 2011. Molecular identification and virulence of three Aeromonas hydrophila isolates cultured from infected channel catfish during a disease outbreak in west Alabama (USA) in 2009. Dis. Aquat. Organ., 94: 249-253. DOI: $10.3354 /$ dao02332

Sasidharan, N.K., S.R. Sreekala, J. Jacob and B. Nambisan, 2014. In vitro synergistic effect of curcumin in combination with third generation cephalosporins against bacteria associated with infectious diarrhea. Biomed Res Int. DOI: 10.1155/2014/561456
Secombes, C.J., T. Wang and S. Bird, 2011. The interleukins of fish. Develop. Comparat. Immunol., 35: 1336-1345.

Tekedar, H.C., G.C. Waldbieser, A. Karsi, M.R. Liles and M.J. Griffin et al., 2013. Complete genome sequence of a channel catfish epidemic isolate, Aeromonas hydrophila strain ML09-119. Genome Announc, 1: 5 e00755-13. DOI: 10.1128 /genomeA.00755-13

Yates, A., C. Bergmann, J.L. Van Hemmen, J. Stark and R. Callard, 2000. Cytokine-modulated regulation of helper T cell populations. J. Theor. Biol., 206: 539-560. DOI: $10.1006 /$ jtbi.2000.2147 